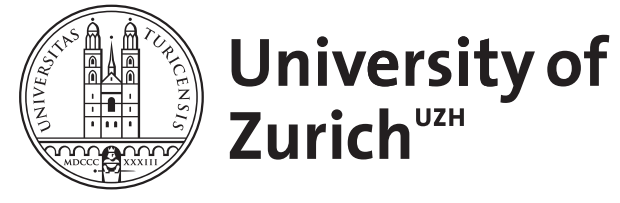

\title{
Helicobacter pylori-Infektion
}

Fried, M

DOI: https://doi.org/10.1007/s11377-006-0051-8

Posted at the Zurich Open Repository and Archive, University of Zurich ZORA URL: https://doi.org/10.5167/uzh-156231

Journal Article

Published Version

Originally published at:

Fried, M (2006). Helicobacter pylori-Infektion. Der Gastroenterologe, 1(4):251-252.

DOI: https://doi.org/10.1007/s11377-006-0051-8 
Gastroenterologe 2006 1 1:251-252 DOI 10.1007/s11377-006-0051-8

Online publiziert: 21. Oktober 2006

๑) Springer Medizin Verlag 2006

M. Fried

Abteilung Gastroenterologie und Hepatologie, Universitätsspital Zürich

\section{Helicobacter pylori-Infektion}

den kritisch gewichtet und einander gegenübergestellt. Die große Zahl der Testverfahren zeigt, dass keine Methode ideal ist, aber auch dass die Diagnoseverfahren differenziert zum Einsatz kommen sollten. Dabei sind auch Kosten-Nutzen-Gesichtspunkte von Bedeutung. Viel zu wenig wird in der Praxis z.B. die Interaktion mit Medikamenten vor dem Einsatz von $H P$-Testverfahren beachtet, was im Artikel besonders beleuchtet wird. So kann eine Vorbehandlung mit einem Protonenpumpenblocker die Sensitivität des ${ }^{13} \mathrm{C}$-Harnsotff-Atemtests deutlich reduzieren. Einige Testverfahren, wie die molekulargenetischen Methoden, sind möglicherweise in der Zukunft von größerer praktischer Bedeutung, aber noch nicht überall verfügbar, auch ist die bisherige Erfahrung mit diesen Verfahren limitiert.

\section{$>$ Die vorliegenden Artikel fassen das heutige Wissen über die HP-Infektion konzis zusammen und beleuchten auch die noch vorhandenen Grauzonen}

Studien zu gewichten und kritisch zu diskutieren. Das Ziel der vorliegenden Ausgabe von "Der Gastroenterologe“ ist, die Fülle von Daten durch international ausgewiesene Experten zu gewichten und praktisch relevante Schlussfolgerungen für den klinisch tätigen Arzt zu ziehen.

Eine Vielzahl von Methoden zur Diagnostik der HP-Infektion ist entwickelt worden, aber nur wenige Methoden haben sich schließlich in der Klinik durchgesetzt. Dazu zählen v. a. bioptisch-histologische Verfahren wie die Histologie und der Urease-Schnelltest, und die ${ }^{13} \mathrm{C}$-Atemtests. Alle diese Methoden haben ihre Vorund Nachteile, die dem Anwender zur korrekten Interpretation der Ergebnisse bekannt sein sollten. Im entsprechenden $A b-$ schnitt werden die verschiedenen Metho- kationsbehandlung unterzogen. Die Autoren gehen auch auf andere heute wichtige potentielle Indikationen der Eradikationstherapie, wie die chronische Einnahme von nichtsteroidalen Antirheumatika und der unklare Eisenmangel ein, aber auch hier ist der Evidenzgrad für eine Eradikation nach der heutigen Datenlage nicht hoch. Besonders diskutiert wird die HP-Eradikation zur Prophylaxe der Magenkarzinoms, wobei eine strikte Indikation nur bei Vorliegen weitere Faktoren (z.B. atrophische Gastritis, familiäre Anamnese) gegeben ist. Auch hier ist die Diskussion noch nicht abgeschlossen und sicher auch von der Information der Patienten v. a. auch durch die Medien geprägt. So wird der Arzt in der Praxis dem Patient, der eine Krebserkrankung fürchtet, kaum den Wunsch nach einer HP-Eradikationsbehandlung ausschlagen können.

Eine Unzahl von Studien wurde zum Thema der HP-Eradikationstherapie publiziert, die Zahl der Kombinationen ist unübersehbar und in steter Wandlung. Von besonderer Bedeutung ist die Zunahme von Resistenzen gegenüber Standardantibioti$k a$, insbesondere Metronidazol, aber auch vermehrt Clarithromycin. Die Autoren zeigen diese Zusammenhänge auf, die v. a. in den letzten Jahren besser verstanden wurden, aber auch andere entscheidenden Erfolgsfaktoren für die Eradikationstherapie, wie z.B. die Compliance des Patienten und die Dauer der Behandlung, die in den letzten Jahren über die bisher als Standard geltende Wochentherapie bis auf 10-14 Tage verlängert wurde.

In den beiden letzten Kapiteln werden die HP-assoziierten Magentumore behandelt. Das HP wurde von der WHO als Karzinogen anerkannt. Trotzdem bleibt noch heute unklar, zu welchem Zeitpunkt der Infektion eine Eradikation einen noch ent- 
scheidenden Einfluss auf den Verlauf der Infektion hat, insbesondere ob die Behandlung eine Transition in ein Karzinom verhindern kann. Das Magenkarzinom ist weltweit noch immer einer der häufigsten Krebsarten und es ist anzunehmen, dass das HP dabei eine entscheidende pathogenetische Rolle spielt. Trotzdem besteht heute nur bei klar definierten Risikogruppen eine unumstrittene Therapieindikation. Eine Eradikationsbehandlung bei der gesamten HP-infizierten Bevölkerung wäre nach Kosten-Nutzen-Kriterien kaum zu rechtfertigen. Nicht vergessen werden sollte aber, dass präneoplastische Läsionen der Magenmukosa sich unter einer HP-Therapie zurückbilden können. Aber auch hier ist heute die Datenlage aufgrund kontrollierter klinischer Studien beim Menschen noch nicht ausreichend, um eindeutige Empfehlungen auszusprechen. Besonders schwierig ist die Identifizierung von Risikogruppen. So wird z. B. nur bei einem kleinen Teil der Patienten mit Dyspepsie eine Endoskopie durchgeführt, die erlauben würde, eine chronische Korpus-dominante Gastritis und eine intestinale Metaplasie, beides Risikofaktoren zur Entwicklung eines Magenkarzinoms, endoskopisch-histologisch zu sichern.

Das MALT-Lymphom ist eine zwar relativ seltene tumoröse Erkrankung des $\mathrm{Ma}$ gens, die HP Infektion spielt hier aber eine besonders prominente pathogenetische Rolle. Eine HP-Eradikation ist hier mit sehr guten Erfolgsaussichten indiziert. In den letzten Jahren haben neuere Untersuchungen gezeigt, dass Residuen des Lymphoms nach einer erfolgreichen HP Eradikation nicht unbedingt eine weitere Eskalation der Therapie mit Operation und Bestrahlung erfordern. Dies ist - beide der Seltenheit der Erkrankung verständlich-vorerst noch auf Beobachtungen bei relativ kleinen Patientenserien begründet. Langzeituntersuchungen bei größeren Patientenkollektiven werden dieses Vorgehen in der Zukunft besser abstützen.

Die vorliegende Serie von Artikeln fasst das heutige Wissen der HP-Infektion konzis zusammen und beleuchtet auch die heutigen Grauzonen unseres Wissens. Die Autoren beschränken sich jedoch nicht aufeine Diskussion der Datenlage sondern schließen jeweils mit eindeutigen und klaren Empfehlungen für die Praxis. Dies macht die Bei- träge zu einem verlässlichen Leitfaden für den Kliniker ohne wichtige Hintergrundinformationen zu vernachlässigen. Dafür ist den Autoren zu danken, die hier eine hervorragende medizinisch publizistische Arbeit geleistet haben. Die HP-Infektion ist auch Jahrzehnte nach der Entdeckung ihrer Bedeutung Gegenstand intensiver Forschung. Die Autoren haben mit Ausblicken auf die künftige Entwicklung dieses wichtigen Gebietes gezeigt, dass wir hier auch weiterhin spannende Ergebnisse erwarten können.

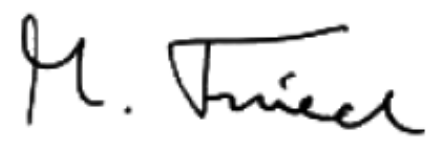

M. Fried, Zürich, Schweiz

\section{Korrespondierender Autor}

Prof. Dr. M. Fried

Abteilung Gastroenterologie und Hepatologie, Universitätsspital Zürich, Rämistraße 100, 8091 Zürich, Schweiz michael.fried@usz.ch

\section{Ileus - altes Problem im neuen Look?}

Aufgrund fehlender zuverlässiger Zahlen zur Inzidenz des lleus ist auch heute noch unklar, wie häufig diese Erkrankung zu einer stationären Krankenhausbehandlung führt. Es kann jedoch davon ausgegangen werden, dass ein lleus bei etwa $10 \%$ aller Patienten vorliegt, die wegen abdomineller Schmerzen notfallmäßig aufgenommen werden.

In der Springer-Fachzeitschrift „Der Chirurg", Ausgabe 10/2006, finden Sie einen qualifizierten Überblick zum Thema lleus. Das Heft enthält Beiträge zu folgenden Themen:

\section{Der Chirurg}

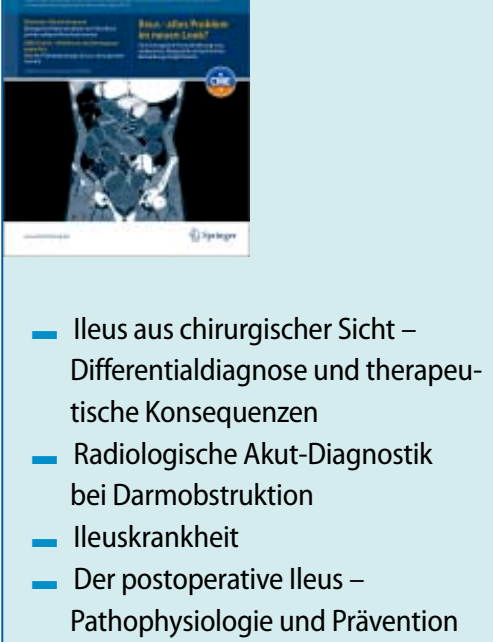

Bestellen Sie diese Ausgabe zum Preis von EUR 27,00 unter folgender Adresse: Springer Distribution Center Kundenservice Zeitschriften Haberstr. 7 69126 Heidelberg Tel.: +49 6221-345-4303 Fax.: +49 6221-345-4229

E-Mail: subscriptions@springer.com

\section{www.DerChirurg.de}

